\title{
Evaluation of BER in LTE System using Various Modulation Techniques over different Modulation Schemes
}

\author{
Parvesh Kumar1, Shalini Bhadola², Kirti Bhatia² \\ 1M.Tech Scholar, ${ }^{2}$ Assistant Professor \\ ${ }^{1,2}$ CSE Department, Sat Kabir Institute of Technology and Management, Bahadurgarh, Haryana, India
}

\begin{abstract}
How to cite this paper: Parvesh Kumar | Shalini Bhadola | Kirti Bhatia "Evaluation of BER in LTE System using Various Modulation Techniques over different Modulation Schemes" Published in International Journal of Trend in Scientific Research and Development

(ijtsrd), ISSN: 2456-

6470, Volume-3 |

Issue-3, April 2019, pp.1325-1329, URL: https://www.ijtsrd. com/papers/ijtsrd2 3315.pdf

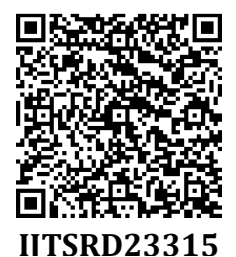

Copyright (C) 2019 by author(s) and International Journal of Trend in Scientific Research and Development Journal. This is an Open Access article distributed under the terms of the Creative Commons

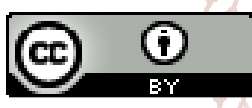
Attribution License (CC BY 4.0) (http://creativecommons.org/licenses/ by $/ 4.0$ )

\section{INTRODUCTION}

Long Term Evolution is the next-generation 4G technology for both Global System for Mobile communication (GSM) and Code Division Multiple Access (CDMA) cellular carriers. Approved in 2008 with download speeds of up to 173 $\mathrm{Mb} / \mathrm{sec}$, LTE was defined by the 3G Partnership Project in the 3GPP Release 8 specification. LTE uses a different air interface and packet structure than the previous $3 \mathrm{G}$ systems, including GSM's UMTS: Wideband CDMA (W-CDMA) and High Speed Packet Access (HSPA), and CDMA's Evolution-Data Optimized (EV-DO). However, it is envisioned that all GSM and CDMA2000 carriers will eventually migrate to LTE to provide an interoperable cellular system worldwide. LTE is a set of enhancements to the UMTS which was introduced in 3GPP Release 8. Much of 3GPP Release 8 focuses on adopting 4G mobile communication technologies, including an all Internet Protocol (IP) flat networking architecture. Along with the Worldwide Interoperability for Microwave Access (WiMAX) 2, the ITU previously designated LTE-A (LTEAdvanced) as the true 4G evolution. In late 2010, the ITU widened its definition to include regular LTE, WiMAX and HSPA+ as bona fide 4G technologies since they are considerably faster than existing $3 G$ networks. LTE uses the Evolved UMTS Terrestrial Radio Access (E-UTRA) air interface, which is based on Orthogonal Frequency Division
Multiple Access (OFDMA) and is a departure from the TDMA used in GSM and the CDMA used in GSM/UMTS and CDMA2000. In addition, LTE is based entirely on IP packets, and voice travels over IP (VoIP). The IP part of LTE is called "Evolved Packet System" (EPS), which was previously called "System Architecture Evolution" (SAE).Although the LTE is often marketed as 4G, first-release LTE does not fully comply with the International Mobile Telecommunications (IMT) Advanced $4 \mathrm{G}$ requirements. The pre- $4 \mathrm{G}$ standard is a step toward LTE Advanced, a 4G standard of radio technologies designed to increase the capacity and speed of mobile telephone networks. LTE Advanced is backwards compatible with LTE and uses the same frequency bands, while LTE is not backwards compatible with $3 \mathrm{G}$ systems.

The organization of present paper is as follow. Section II presents overview of OFDM. Section III describes the methodology used for proposed work as in this paper modulation technique is used. Result analysis is presented in section IV following the concluding remarks in section V.

\section{OFDM Structure}

The incoming serial data is first converted from serial to parallel and grouped into $\mathrm{x}$ bitseach to form a complex 
number. The complex numbers are modulated in a baseband fashion by the IFFT. and converted back to serial data for transmission. A guard interval is inserted between symbols to avoid intersymbol interference (ISI) caused by multipath distortion. The discrete symbols are converted to analog and low pass filtered for $\mathrm{RF}$ up-conversion. The receiver performs the inverse process of the transmitter. One tap equalizer is used to correct channel distortion. The tap coefficients of the filter are calculated based on channel information.
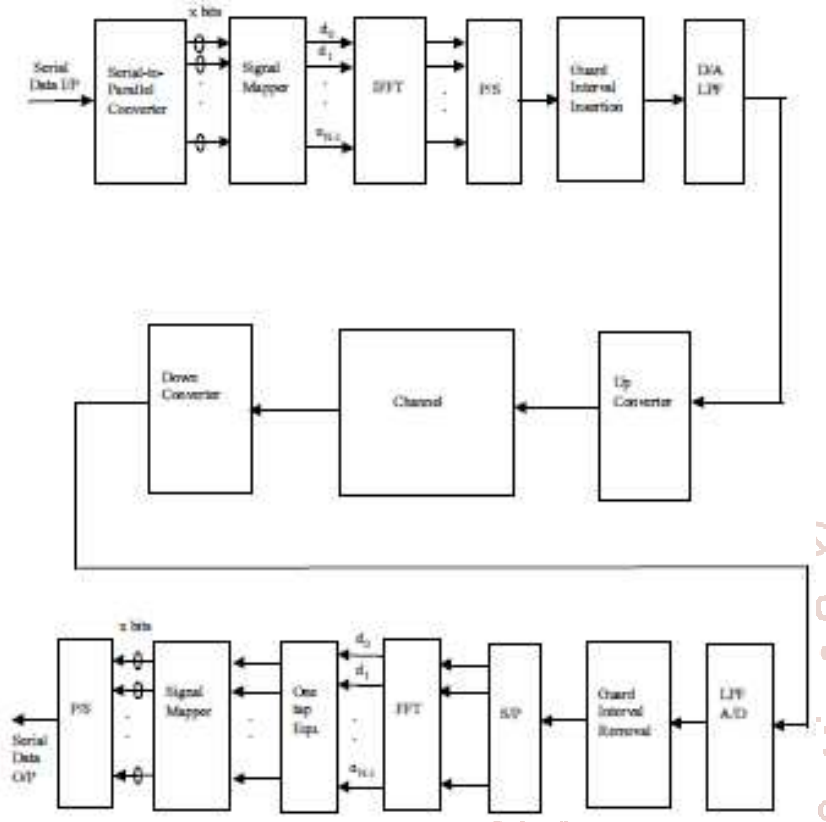

Fig1 OFDM Structure
$\overline{R_{p_{j}}}=\sum_{j=1}^{N_{F}} \bar{P} \bar{H}_{P_{j}}+\bar{N}_{p_{j}}$
$\overline{R_{j}}=\sum_{j=1}^{N_{r}} \overline{S H}_{j} \| \bar{N}_{j}$

where and represent the received pilot and data signal at the $j^{\text {th }}$ antenna respectively and are the pilot and data signals respectively, , and, represent the channel parameters and the white Gaussian noise between the two transmit antennas and the $N_{r}$ receive antennas for pilot and data signals respectively. The pilot elements of (4.2) can be expressed as: $\bar{R}_{p j}=\left[R_{p_{j k_{p}}}(n) R_{p_{j k_{p}}}(n+1)\right]^{T}$

$\bar{H}_{p_{j}}=\left[\begin{array}{c}H_{p_{j, k_{p}}}(n) \\ H_{p_{j, k_{p}}}(n+1)\end{array}\right]$

$\bar{P}=\left[\begin{array}{cc}P_{1, k_{p}}(n) & P_{2, k_{p}}(n) \\ -P_{i, k_{p}}^{*}(n) & P_{1, k_{p}}^{*}(n)\end{array}\right] \bar{N}_{p_{j}}=\left[\begin{array}{c}N_{p_{j, k}}(n) \\ N_{p_{j, k_{p}}}(n+1)\end{array}\right]$

where $k_{p}=0,1, \ldots, N p-1$. While the data elements are given as: $\bar{R}_{j}=\left[R_{j k}(n) F_{j k}(n+1)\right]^{T}$

$\bar{H}_{j}=\left[\begin{array}{c}H_{1, j k}(n) \\ H_{2 j k}(n+1)\end{array}\right]$

$\bar{S}=\left[\begin{array}{cc}S_{1 k}(n) & S_{2 k}(n) \\ -S_{2 k}^{*}(n) & S_{1 k}^{*}(n)\end{array}\right] \bar{N}_{P_{j}}=\left[\begin{array}{c}N_{1, k}(n) \\ N_{2, k}(n+1)\end{array}\right]$

where $\mathrm{k}=0,1, \ldots, \mathrm{Ns}-1$.

Transmitted pilot and data signals are encoded in space, time and frequency as described in Fig 4-4. Therefore, P1(n) and P2(n) transmitted in MIMO symbol $n$ and P1 $(n+1)$ and $\mathrm{P} 2(\mathrm{n}+1)$ transmitted during the second MIMO symbol $n+1$ can be expressed by (4.5).

Based on (4.1), received pilot and data subcarriers signal rch and after FFT can be expressed as:

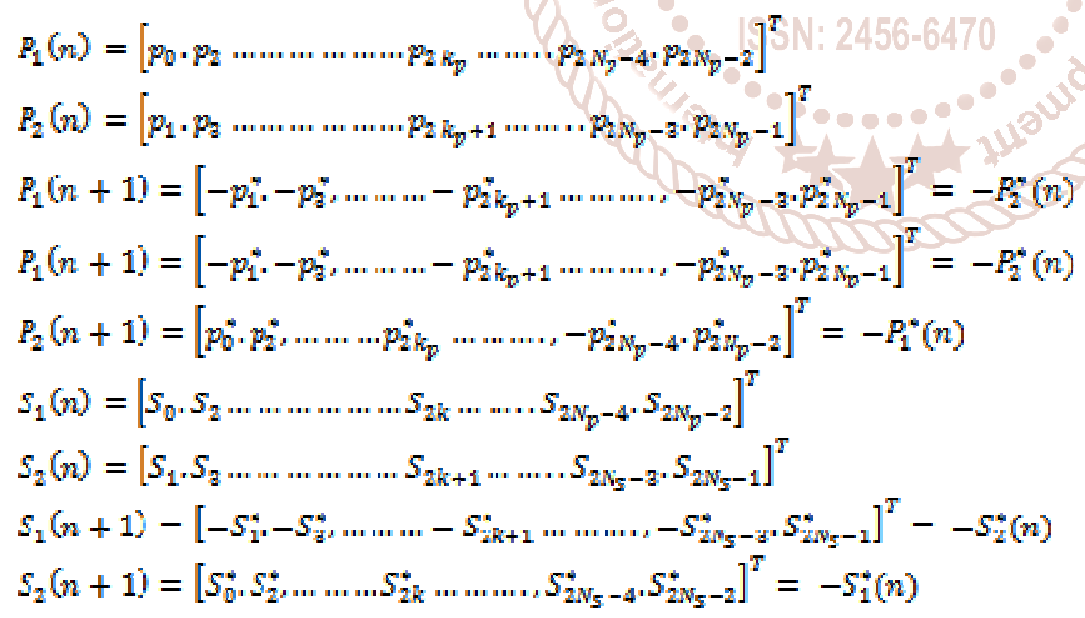

The received vector and at MIMO symbols $n$ and $n+1$ can be expressed as:

$$
\begin{aligned}
& R_{p j}(n)=\left[r p_{j, 0}, r p_{j, 2}, \ldots \ldots \ldots \ldots r p_{j, 2 N_{p}-4, r p_{j, 2 N_{p}}-2}\right]^{T} \\
& R_{p j}(n+1)=\left[r p_{i, 1}, r p_{i, z}, \ldots \ldots \ldots \ldots r p_{i}, 2 N_{p}-2, r p_{i, 2 N_{p}-1}\right]^{T} \\
& R_{j}(n)=\left[\eta_{j,}, \eta_{2}, \ldots \ldots \ldots, \ldots, \eta_{2 N_{z}-4}, \eta_{2, N_{z}-2}\right]^{T}
\end{aligned}
$$

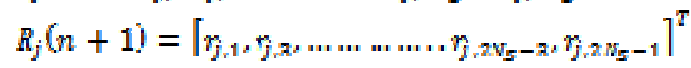

At the receiver side, as stated in Section 4.3, channel parameters of two consecutive MIMO symbols are assumed constant therefore and the receiver uses the pilot sequence in (5.2) to estimate the channel coefficients using the minimum mean square error (MMSE) as i

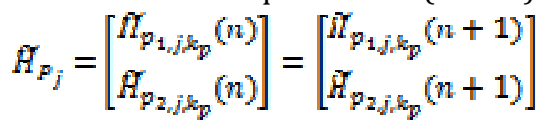

Once channel parameters are evaluated, the receiver can detect the upper and lower data symbols of the adjacent data subcarrier. Using in (4.7) and in (4.4), the receiver constructs a new channel matrix and a new receive matrix given as

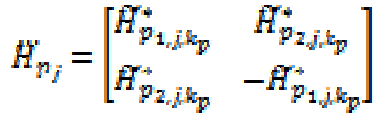


$\tilde{R}_{j}=\left[R_{j, k}(n) R_{j, k}^{*}(n+1)\right]^{T}$

The receiver uses the constructed channel matrix $H_{p_{j}}$ and the constructed received data vector $k_{j}$ for the combining scheme. Assuming that channel parameters of two adjacent subcarriers and two consecutive

\section{Frame Work for Implementation}

The main objectives of research work areto Study and analyze various technologies such as LTE, MIMO OFDM, Wireless Channel Modeling, Channel Fading and Modulation Techniques.To design a LTE system that supports various Modulation techniques and which can be tested using various Fading Channels. To evaluate the performance of proposed LTE system with various evaluation metrics such as BER and SNR.

Consider a complex tone signal: $\mathrm{x}(\mathrm{t})=e^{2 \pi f \mathrm{t}}$

with a period $\mathrm{T}$. The peak value of the signal is: $\max \left[\mathrm{x}(\mathrm{t}) \mathrm{x}^{*}(\mathrm{t})\right]=\max \left[e^{2 \pi f t} \epsilon^{-2 \pi f t}\right]=\max \left[e^{\theta}\right]=1$

The mean square value of the signal is: $\mathrm{E}\left[\mathrm{x}(\mathrm{t}) \mathrm{x}^{*}(\mathrm{t})\right]=\mathrm{E}\left[e^{2 \pi f t} e^{-2 \pi f \mathrm{t}}\right]=1$

This gives us a PAPR of $0 \mathrm{~dB}$. Consider that an OFDM time signal is made of $K$ complex tones (usually called subcarriers). Our signal can be represented by the following formula

$\mathrm{x}(\mathrm{t})=\Sigma_{0}^{K-1} c_{k} e^{\frac{j 2 \pi k x}{T}}$

Trend in Researc-QAM is represented in fig 3.

For simplicity, let's assume $a_{k}=1$ for any $k$. In this scenario, lopment: the peak value of the signal is: $\max \left[\mathrm{x}(\mathrm{t}) \mathrm{x}^{*}(\mathrm{t})\right]=\max$

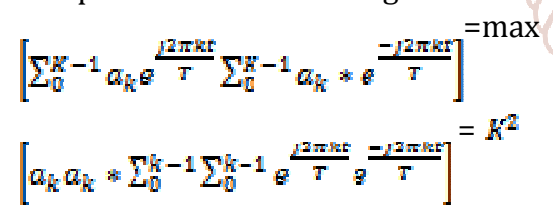

The mean square value of the signal is:

$\mathrm{E}\left[\mathrm{x}(\mathrm{t}) \mathrm{x}^{*}(\mathrm{t})\right]=\mathrm{E}\left[\sum_{0}^{K-1} \alpha_{k} e^{\frac{12 \pi k t}{T}} \sum_{0}^{K-1} a_{k} * g^{\frac{-j 2 \pi k t}{\mathrm{~T}}}\right]=\mathrm{E}$

$\left[a_{k} \alpha_{k} * \sum_{0}^{k-1} \sum_{0}^{k-1} e^{\frac{j 2 \pi k t}{T}} e^{\frac{-12 \pi k t}{T}}\right]=\mathrm{K}$

Constellation diagram of different modulation technique is given as

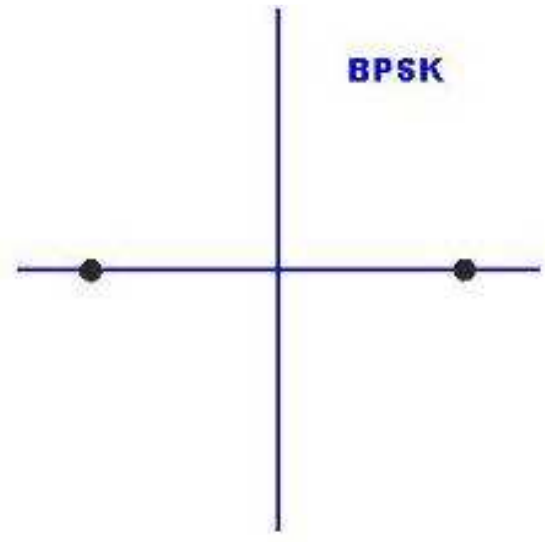

Fig 2 Constellation Diagram of BPSK
The simplest form of PSK is binary phase-shift keying(BPSK), where $\mathrm{N}=1$ and $\mathrm{M}=2$. Therefore, with BPSK, two phases (21 $=2$ ) are possible for thecarrier.One phase represents a logic 1 , and the other phase representsa logic 0 . As the input digital signal changes state (i.e., from a 1 to a 0 or from a 0 to a 1), the phase of the output carrier shiftsbetween two angles that are separated by $180^{\circ}$. Constellations diagram of BPSK is represented in fig 2.

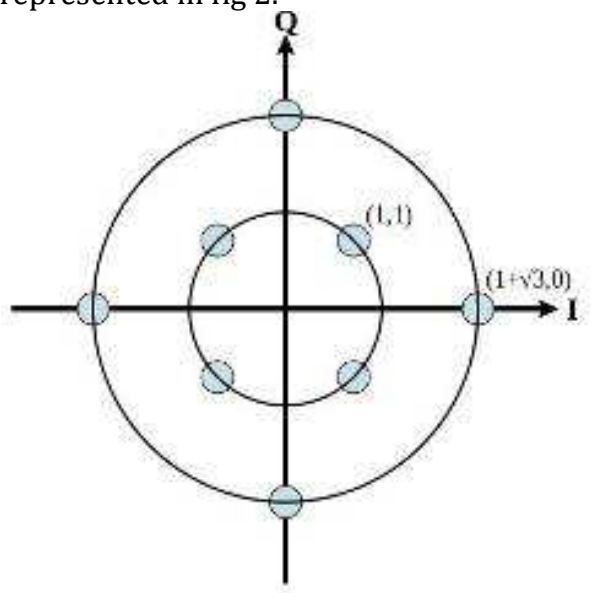

Fig 3 Constellation Diagram of 8 - QAM
8-QAM is an $\mathrm{M}$-ary encoding technique where $\mathrm{M}=8$. Unlike 8-PSK, the output signal from an 8-QAM modulator is not a constant-amplitude signal. The outputs from the I and Q channel product modulators are combined in the linear summer and produce a modulated output of summer output $=-0.541$ sin_ct. -0.541 cos_ct. $=0.765 \sin \left(\cos -135^{\circ}\right)$. For the remaining tribit codes $(001,010,011,100,101,110$, and 111 ), the procedure is the same. Constellations diagram of 8

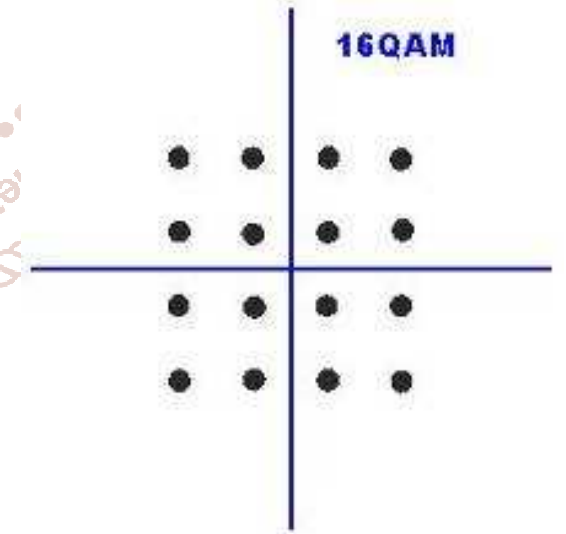

Fig 4 Constellation Diagram of 16 - QAM

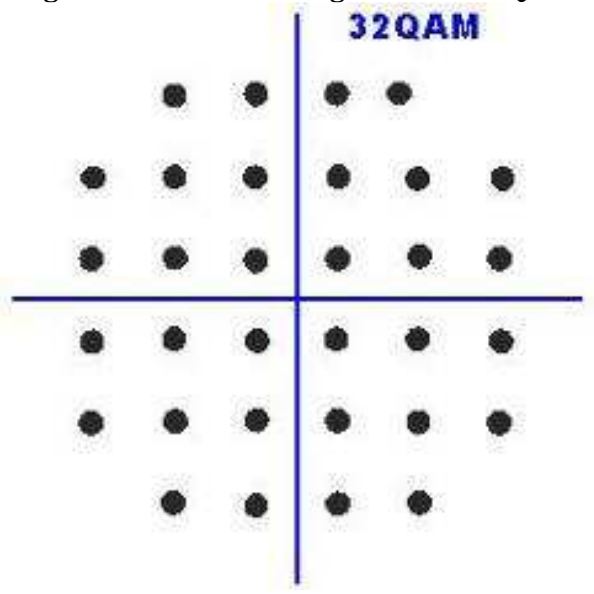

Fig 5 Constellation Diagram of 32 - QAM 
32 - QAM constellation is explain in the fig 5 . There is 32 points each are $45^{\circ}, 135^{\circ}$ respectively.

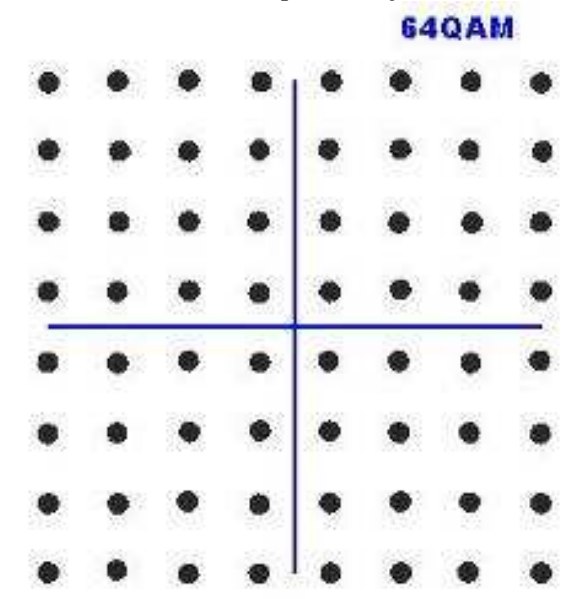

Fig 6 Constellation Diagram of 64 - QAM

64 - QAM constellation is explain in the fig 5 . There is 64 points.eachpoints are parallel align to each other.

\section{Result Analysis}

In this paper, main focus on the bit error rate of different modulation technique.Table 1 represent the parameter used for evaluation of LTE Bit error rate.

Table 1 Parameters for Evaluation of LTE BER

\begin{tabular}{|c|c|}
\hline Parameter Name & Description \\
\hline Transport Block Size & Size of transport block \\
\hline AvailablePDSCHBits & $\begin{array}{c}\text { Size of coded transport } \\
\text { block after rate matching } \\
\text { (codeword size) }\end{array}$ \\
\hline Modulation & $\begin{array}{l}\text { Modulation scheme, one of } \\
\text { \{'QPSK', '16QAM', '32-QAM' } \\
\text { '64-QAM'\} }\end{array}$ \\
\hline SNRRange & $\mathrm{Eb} /$ No range in $\mathrm{dB}$ \\
\hline RVSeq & $\begin{array}{l}\text { Redundancy version } \\
\text { indicators sequence }\end{array}$ \\
\hline NTurboDecIts & $\begin{array}{c}\text { Number of turbo decoder } \\
\text { iteration cycles }\end{array}$ \\
\hline OverlayGraphs & $\begin{array}{c}\text { Holds the previous graphs } \\
\text { when checked, thus } \\
\text { overlays new curve on } \\
\text { previously drawn curves }\end{array}$ \\
\hline
\end{tabular}

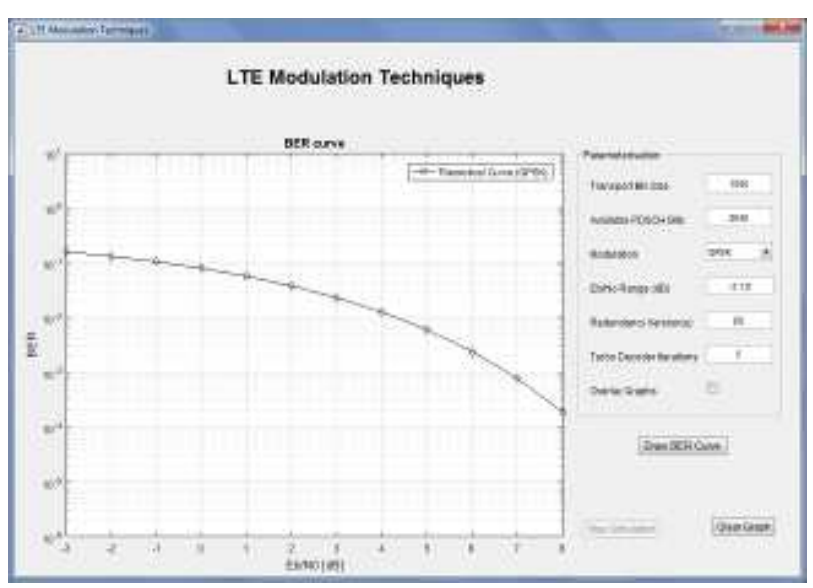

Fig 3 initial Screen with Theoretical LTE BER Evaluation
Fig 3 represents theoretical LTE bit rate evaluation. A random stream of bits the size of the desired transport block undergoes Downlink Shared Channel (DL-SCH) coding to rate match the transport block to the available PDSCH bits. Scrambling, modulation, pre-coding and layer mapping are then applied to form the complex PDSCH symbols. AWGN is added to these symbols after which channel decoding and demodulation are performed to recover the transport block. Using the recovered transport block a BER curve is plotted for a given range of SNR values.

If we compare with the previous work, BER is reduce by 1.1 $\mathrm{db}$.

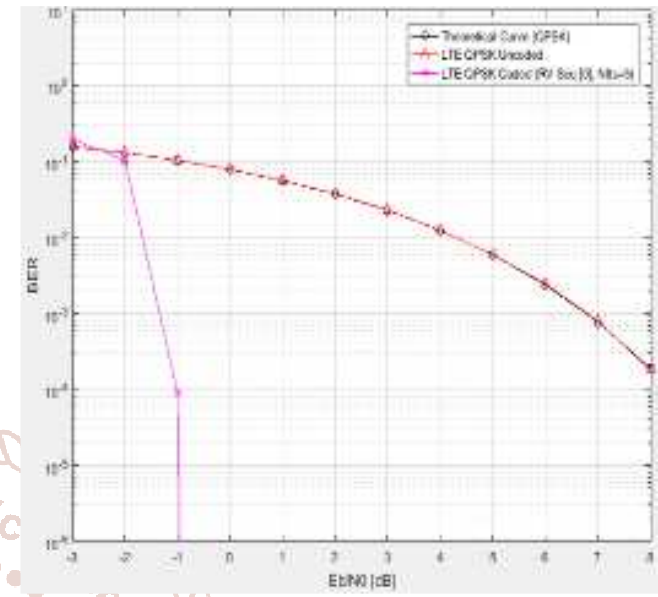

Fig 4 BER Curve for LTE under QPSK Modulation with Uncoded and Coded Bits

Fig 4 represent the bit error rate curve for LTE system.. These are comes for QPSK modulation system. BER for QPSK is minimized with previous work is $1.119 \mathrm{db}$

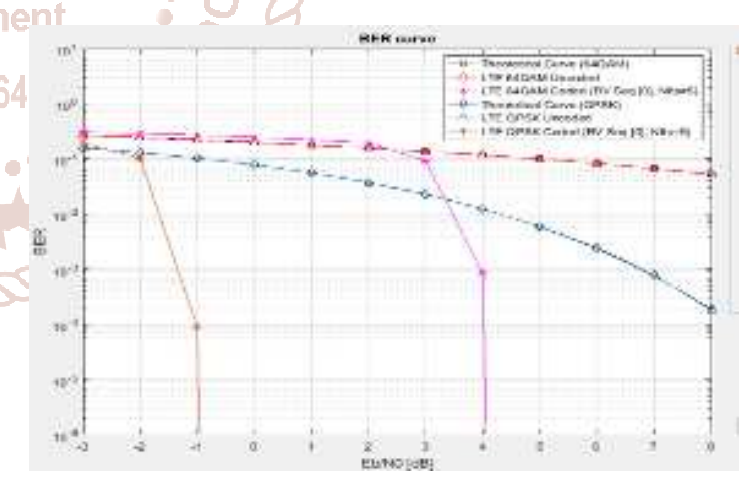

Fig 5 Comparison of QPSK and 64 Modulations over LTE

A comparison is made for QPSK \& 64 bit modulation over the long term evaluation system. As compare to previous research, applied modulation technique is optimized.

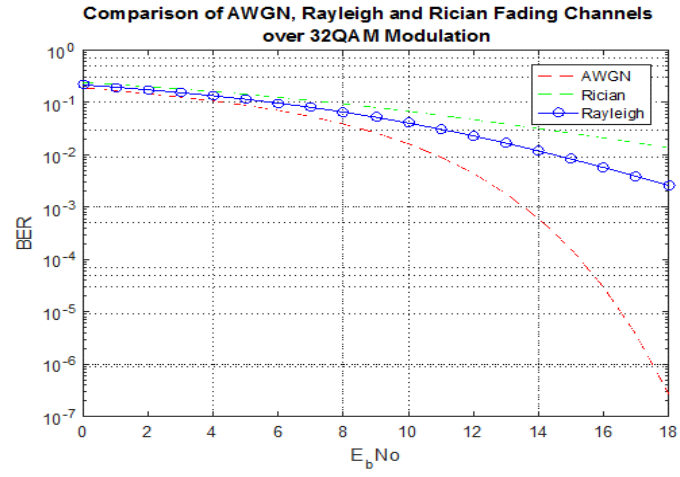

Fig 6Comparison of AWGN, Rayleigh and Racian Fading channel 
Figure 6 represent the comparison between the AWGN, Rayleigh and the racian fading channel. AWGN channel have very less noise as compare to other channel. The proposed channel provides less noise as compare previous work in racian channel

\section{Conclusion}

We evaluated the act of available transmission modes in LTE. However, performance analysis can be done straightforward using evaluation of LTE. The performance of transmission modes are evaluated by calculating probability of Bit Error Rate (BER) versus Signal Noise Ratio (SNR) under the frequently used three wireless channel models (AWGN, Rayleigh and Rician). We will consider the data modulation and data rate to analyze performance that is BER vs. SNR. The evaluation of performance will confirm increase in the coverage area of the physical layer in the LTE devices. The improved a model can describe a fading environment, improved can it be compensated with other signals, so that, on the receiving end, signal is error free.

\section{References:}

[1]. Giri, NimayCh, AnweshaSahoo, J. R. Swain, P. Kumar, A. Nayak, and P. Debogoswami. "Capacity \& performance comparison of SISO and MIMO system for next generation network (NGN)." International Journal of Advanced Research in Computer Engineering \& Technology (IJARCET) Volume 3 (2014).

[2]. Patel, Brijesh Kumar, and JatinAgarwal. "Comparative Study of Bit Error Rate with Channel Estimation in OFDM System for M-ary Different Modulation Techniques." International Journal of Computer Applications 95, no. 8 (2014).

[3]. Lee, Donghun, and Byung Jang Jeong. "Performance analysis of combining space-time block coding and scheduling over arbitrary Nakagami fading channels." IEEE Transactions on Wireless Communications 13, no. 5 (2014): 2540-2551.

[4]. Sarala, B., D. S. Venketeswarlu, B. N. Bhandari, and B. Srinivas. "Discrete Transforms based MC-CDMA PAPR Reduction using MECCT and Bit Error Rate Performance Analysis over Mobile Radio Channels." In Proceedings of International Conference on Recent Trends in Information, Telecommunication and Computing, ITC, pp. 238-247. 2014.

[5]. Kumar, Naresh. "BER analysis of conventional and wavelet based OFDM in LTE using different modulation techniques." In Engineering and Computational Sciences (RAECS), 2014 Recent Advances in, pp. 1-4. IEEE, 2014.

[6]. Huang, Chuan, Rui Zhang, and Shuguang Cui. "Optimal power allocation for outage probability minimization in fading channels with energy harvesting constraints."
IEEE Transactions on Wireless Communications 13, no. 2 (2014): 1074-1087.

[7]. Islam, MdShoriful, Ahmed Al Amin, Shuva Paul, and Intisar Tahmid. "Reliability Checking for Digital Modulation Schemes in 4G-5G Communication system: Comparison between QPSK and QAM Modulation Techniques for Beyond LTE." In Proceedings of the Sixth International Conference on Computer and Communication Technology 2015, pp. 442-446. ACM, 2015.

[8]. Ndujiuba, Charles U., Oluyinka Oni, and Augustus E. Ibhaze. "Comparative Analysis of Digital Modulation Techniques in LTE 4G Systems." Journal of Wireless Networking and Communications 5, no. 2 (2015): 6066.

[9]. Chafii, Marwa, M. LamaranaDiallo, Jacques Palicot, Faouzi Bader, and RémiGribonval. "Adaptive Tone Reservation for better BER Performance in a Frequency Selective Fading Channel." In IEEE VTC2016-Spring. 2016.

[10]. Ghosh, Sutanu. "Performance Evaluation of Different Coding and Modulation Scheme in LTE Using Different Bandwidth and Correlation Levels." Wireless Personal Communications 86, no. 2 (2016): 563578.(CONECCT), 2013 IEEE International Conference on, pp. 1-6. IEEE, 2013.

[11]. Gao, Hui, Chau Yuen, Himal A. Suraweera, and Tiejun Lv. "Multiuser diversity for MIMO-Y channel: Max-min selection and diversity analysis." InCommunications (ICC), 2013 IEEE International Conference on, pp. 57865791. IEEE, 2013.

[12]. Te Chen, Lu Liu, Bo Tu, ZhongZheng, and Weiwei Hu. "High-spatial-diversity imaging receiver using fisheye lens for indoor MIMO VLCs." (2014).

[13]. Wang, Wen-Qin. "MIMO SAR chirp modulation diversity waveform design."IEEE Geoscience and Remote Sensing Letters 11, no. 9 (2014): 1644-1648.

[14]. Meng, Xin, Xiang-Gen Xia, and XiqiGao. "Constantenvelope omni-directional transmission with diversity in massive MIMO systems." In Global Communications Conference (GLOBECOM), 2014 IEEE, pp. 3784-3789. IEEE, 2014.

[15]. Sahrab, Ammar Ali, and Ion Marghescu. "MIMO-OFDM: Maximum diversity using maximum likelihood detector." In Communications (COMM), 2014 10th International Conference on, pp. 1-4. IEEE, 2014.

[16]. Tanbourgi, Ralph, Harpreet S. Dhillon, and Friedrich K. Jondral. "Analysis of joint transmit-receive diversity in downlink MIMO heterogeneous cellular networks." IEEE Transactions on Wireless Communications 14, no. 12 (2015): 6695-6709. 